\title{
Novel Fuzzy-Modeling-Based Adaptive Synchronization of Nonlinear Dynamic Systems
}

\author{
Shih-Yu Li, ${ }^{1,2}$ Chin-Sheng Chen, ${ }^{1}$ Lap-Mou Tam, ${ }^{2,3}$ and Shun-Hung Tsai ${ }^{1}$ \\ ${ }^{1}$ Graduate Institute of Automation Technology, National Taipei University of Technology, Taipei, Taiwan \\ ${ }^{2}$ Institute for the Development and Quality, Macau \\ ${ }^{3}$ Department of Electromechanical Engineering, Faculty of Science and Technology, University of Macau, Macau
}

Correspondence should be addressed to Shun-Hung Tsai; shtsai@mail.ntut.edu.tw

Received 19 July 2016; Accepted 19 January 2017; Published 7 March 2017

Academic Editor: Driss Boutat

Copyright (C) 2017 Shih-Yu Li et al. This is an open access article distributed under the Creative Commons Attribution License, which permits unrestricted use, distribution, and reproduction in any medium, provided the original work is properly cited.

\begin{abstract}
In this paper, a novel fuzzy-model-based adaptive synchronization scheme and its fuzzy update laws of parameters are proposed to address the adaptive synchronization problem. The proposed fuzzy controller does not share the same premise of fuzzy system, and the numbers of fuzzy controllers is reduced effectively through the novel modeling strategy. In addition, based on the adaptive synchronization scheme, the error dynamic system can be guaranteed to be asymptotically stable and the true values of unknown parameters can be obtained. Two identical complicated dynamic systems, Mathieu-Van der pol system (M-V system) with uncertainties, are illustrated for numerical simulation example to show the effectiveness and feasibility of the proposed novel adaptive control strategy.
\end{abstract}

\section{Introduction}

Since Professor Zadeh firstly proposed the fuzzy logic concept in 1988 [1], fuzzy logic has received much attention and served as an effective tool for nonlinear analysis and fuzzy control system [2-5]. Among various kinds of fuzzy methods, Takagi-Sugeno (T-S) fuzzy system [6] is widely accepted as a useful tool for design and analysis of fuzzy system, such as sliding mode control $[7,8]$, time-vary delay $[9,10]$, Robust H-infinity stabilization [11, 12], polynomial fuzzy model approach [13], and other applications on fuzzy systems $[14,15]$. T-S fuzzy model is sensitive to the numbers of nonlinear terms-the numbers of fuzzy rules are generated according to the numbers of nonlinear terms in a nonlinear dynamic system. Therefore, if there are lots of nonlinear terms in a dynamic system, the modeling process should be complicated and numerous modeling materials are required. Large amounts of linear subsystems with their membership functions are needed to express the original dynamic system, and numerous sets of fuzzy controllers are necessary for synchronization problem.
Based on the concept of classical T-S fuzzy system, Ge and $\mathrm{Li}$ [16] presented an alternative fuzzy modeling strategy in the end of 2011, which is focusing on modeling each nonlinear equation of nonlinear dynamic systems. This new model is effectively raising the modeling efficiency, simplifying the modeling process, and reducing the numbers of controllers during system control. Further, Li et al. [17] contributed a new concept of fuzzy modeling in 2015, nonlinear terms groups, to improve the Ge-Li fuzzy model-the final output of the proposed fuzzy model can be composed of only two linear subsystems and two sets of membership function matrices. This new modeling approach leads the final output of the fuzzy system to be a "standard form" with fixed numbers of linear subsystems and its corresponding membership function matrices. In other words, through the novel model, two different nonlinear dynamic systems with distinct structures can be simply modeled as a unified form and the controller design can be more flexible.

In this article, an extended study of the novel fuzzy model on parameter mismatch is proposed. Since the exact knowledge of system structure and parameters are always 
unknown in many application cases and those parameters may change from time to time, a novel fuzzy-model-based adaptive synchronization scheme and its novel adaptation laws of parameters with fuzzy IF-THEN rules are further derived to address the problem of systems synchronization with parameters mismatch.

The contents of this paper are described as follows. The theory of the novel fuzzy system and the fuzzy adaptive synchronization scheme are introduced in Section 2. Simulation results of fuzzy synchronization are proposed in Section 3. Finally, conclusions are given in Section 4.

\section{Fuzzy Adaptive Synchronization Scheme}

In this section, a novel fuzzy-model-based adaptive synchronization scheme is provided, which introduces the design of fuzzy controllers and the derivation of adaptation laws of parameters with fuzzy IF-THEN rules. The kernel modeling strategy of the proposed fuzzy model [17] is to package all nonlinear terms as a group in each equation and transfer the original dynamic system to be two linear subequations with their corresponding membership function matrices. The final output of this fuzzy system representing a nonlinear dynamic system with n-states can be simply described as follows:

$$
\dot{\mathbf{Y}}(\mathbf{t})=\sum_{i=1}^{p} \widetilde{\mathbf{M}}_{i}\left(\mathbf{A}_{i} \mathbf{Y}(\mathbf{t})+\mathbf{B}_{i}\right)
$$

where $\mathbf{Y}(\mathbf{t}) \in \boldsymbol{R}^{n \times 1}$ is the system-state vector and $\mathbf{A}_{i} \in \boldsymbol{R}^{n \times n}$ and $\mathbf{B}_{i} \in \boldsymbol{R}^{n \times n}$ are system coefficient matrices, where $\mathbf{B}_{i}$ includes some constants through modeling transform. $p=2$, which represents the total numbers of linear subsystems and membership functions matrices through the novel modeling strategy. In addition, $\widetilde{\mathbf{M}}_{i} \in \boldsymbol{R}^{n \times n}$ are membership functions matrices that can be described as follows:

$$
\widetilde{\mathbf{M}}_{i}=\left[\begin{array}{cccc}
M_{j i} & 0 & 0 & 0 \\
0 & M_{j i} & 0 & 0 \\
0 & 0 & \ddots & 0 \\
0 & 0 & 0 & M_{j i}
\end{array}\right], \quad j=1,2, \ldots, n ; i=1,2,
$$

where $\widetilde{\mathbf{M}}_{1}+\widetilde{\mathbf{M}}_{2}$ is identity matrix and $\sum_{j=1}^{2} M_{i j}=1$.

Consider the following two identical $n$-orders fuzzy systems in (3) and (4), which are regarded as the master system with all unknown parameters and the slave system, respectively.

Master system:

$$
\dot{\mathbf{Y}}(\mathbf{t})=\sum_{i=1}^{p} \widetilde{\mathbf{M}}_{i}\left(\mathbf{A}_{i} \mathbf{Y}(\mathbf{t})+\mathbf{B}_{i}\right)
$$

Slave system:

$$
\dot{\mathbf{X}}(\mathbf{t})=\sum_{i=1}^{p} \widetilde{\mathbf{N}}_{i}\left(\widehat{\mathbf{A}}_{i} \mathbf{X}(\mathbf{t})+\widehat{\mathbf{B}}_{i}\right)+\mathbf{U}(\mathbf{t}),
$$

where $\mathbf{A}_{i} \in \boldsymbol{R}^{n \times n}$ and $\mathbf{B}_{i} \in \boldsymbol{R}^{n \times n}$ are the coefficient matrices with some unknown parameters; $\widehat{\mathbf{A}}_{i} \in \boldsymbol{R}^{n \times n}$ and $\widehat{\mathbf{B}}_{i} \in \boldsymbol{R}^{n \times n}$ are the coefficient matrices with some estimate parameters; $\mathbf{U}(\mathbf{t}) \in \boldsymbol{R}^{n \times 1}$ is designed as the fuzzy adaptive control inputs outside the fuzzy modeling and does not share the same membership functions of fuzzy-model-based system. Here, we define the error of states $\mathbf{e}(\mathbf{t}) \in \boldsymbol{R}^{n \times 1}$ and the error of unknown and estimated parameters $\widetilde{\mathbf{A}}_{i} \in \boldsymbol{R}^{n \times n}$ and $\widetilde{\mathbf{B}}_{i} \in$ $\boldsymbol{R}^{n \times n}$ as follows:

$$
\begin{aligned}
\mathbf{e}(\mathbf{t}) & =\mathbf{Y}(\mathbf{t})-\mathbf{X}(\mathbf{t}) \\
\widetilde{\mathbf{A}}_{i} & =\mathbf{A}_{i}-\widehat{\mathbf{A}}_{i} \\
\widetilde{\mathbf{B}}_{i} & =\mathbf{B}_{i}-\widehat{\mathbf{B}}_{i} .
\end{aligned}
$$

Then we have the following error dynamics description:

$$
\begin{aligned}
\dot{\mathbf{e}} & =\dot{\mathbf{Y}}-\dot{\mathbf{X}} \\
& =\sum_{i=1}^{2} \widetilde{\mathbf{M}}_{i}\left(\mathbf{A}_{i} \mathbf{Y}(\mathbf{t})+\mathbf{B}_{i}\right)-\sum_{i=1}^{2} \widetilde{\mathbf{N}}_{i}\left(\widehat{\mathbf{A}}_{i} \mathbf{X}(\mathbf{t})+\widehat{\mathbf{B}}_{i}\right)-\mathbf{U}(\mathbf{t})
\end{aligned}
$$

Since $\widetilde{\mathbf{M}}_{i} \neq \widetilde{\mathbf{N}}_{i}$, the error dynamic system is rearranged to be another form:

$$
\begin{aligned}
\dot{\mathbf{e}}= & \sum_{i=1}^{2} \widetilde{\mathbf{M}}_{i}\left(\mathbf{A}_{i} \mathbf{Y}(\mathbf{t})+\mathbf{B}_{i}\right)-\sum_{i=1}^{2} \widetilde{\mathbf{N}}_{i}\left(\widehat{\mathbf{A}}_{i} \mathbf{X}(\mathbf{t})+\widehat{\mathbf{B}}_{i}\right) \\
& -\sum_{i=1}^{2} \widetilde{\mathbf{M}}_{i}\left(\mathbf{A}_{i} \mathbf{X}(\mathbf{t})+\widehat{\mathbf{B}}_{i}\right) \\
& +\sum_{i=1}^{2} \widetilde{\mathbf{M}}_{i}\left(\mathbf{A}_{i} \mathbf{X}(\mathbf{t})+\widehat{\mathbf{B}}_{i}\right)-\mathbf{U}(\mathbf{t}) \\
= & \sum_{i=1}^{2} \widetilde{\mathbf{M}}_{i}\left(\widetilde{\mathbf{A}}_{i} \mathbf{Y}(\mathbf{t})+\widetilde{\mathbf{B}}_{i}+\widehat{\mathbf{A}}_{i} \mathbf{e}(\mathbf{t})\right) \\
& -\sum_{i=1}^{2}\left(\widetilde{\mathbf{M}}_{i}-\widetilde{\mathbf{N}}_{i}\right)\left(\widehat{\mathbf{A}}_{i} \mathbf{X}(\mathbf{t})+\widehat{\mathbf{B}}_{i}\right)-\mathbf{U}(\mathbf{t}) .
\end{aligned}
$$

According to (7), we can design the fuzzy controllers $\mathbf{U}(\mathbf{t})$ as follows:

$$
\mathbf{U}(\mathbf{t})=\sum_{i=1}^{2}\left(\widetilde{\mathbf{M}}_{i}-\widetilde{\mathbf{N}}_{i}\right)\left(\widehat{\mathbf{A}}_{i} \mathbf{X}(\mathbf{t})+\widehat{\mathbf{B}}_{i}\right)+\sum_{i=1}^{2} \widetilde{\mathbf{M}}_{i}\left(\mathbf{K}_{i} \mathbf{e}(\mathbf{t})\right),
$$

where $\mathbf{K}_{i} \in \boldsymbol{R}^{n \times n}$ and $\mathbf{K}_{i}=\widehat{\mathbf{A}}_{i}-\mathbf{G} ; \mathbf{G} \in \boldsymbol{R}^{n \times n}$ is designed as a positive-definite matrix. Substituting the designed fuzzy controllers into (7), we have the following simple form of error dynamic system:

$$
\dot{\mathbf{e}}(\mathbf{t})=\sum_{i=1}^{2} \widetilde{\mathbf{M}}_{i}\left(\widetilde{\mathbf{A}}_{i} \mathbf{Y}(\mathbf{t})+\widetilde{\mathbf{B}}_{i}\right)-\mathbf{G e}(\mathbf{t}) .
$$

Consider the following candidate Lyapunov function:

$$
\mathbf{V}\left(\mathbf{e}(\mathbf{t}), \widetilde{\mathbf{a}}_{i}\right)=\frac{1}{2}\left(\mathbf{e}(\mathbf{t})^{2}+\sum_{i=1}^{m}\left(\mathbf{F}_{i} \widetilde{\mathbf{a}}_{i}^{2}\right)\right),
$$


where $\widetilde{\mathbf{a}}_{i}$ are the error of unknown and estimated parameters, $m$ is the total number of unknown parameters, and $\mathbf{F}_{i}$ refers to the adaptation gains where $\mathbf{F}_{i}>0$; in this paper, all values of $\mathbf{F}_{i}$ are set to be $\mathbf{F}_{i}=1$. Particularly, in this article, a set of innovative fuzzy adaptation law of parameters based on fuzzy IF-THEN rules is provided to further address the mismatch of parameters, and the design procedure is given below.

2.1. Fuzzy Adaptation Law of Parameters. If $\widetilde{a}_{k}$ is the unknown parameter in the $j$ th equation, then we have

$$
\begin{aligned}
& \text { Rule i: IF } N_{j}(t) \text { is } M_{j i} \text {, THEN } \dot{\tilde{a}}_{k}(t) \\
& \quad=f\left(x(t), y(t), W_{j}\right),
\end{aligned}
$$

where $i=1-2, j=1,2, \ldots, n, n$ is the number of states, $N_{j}(t) \in \mathfrak{R}^{1 \times 1}$ is the nonlinear terms group in the $j$ th nonlinear equation, and $M_{j i} \in \mathfrak{R}^{1 \times 1}$ is the membership function corresponding to the fuzzy Rule $i$. Through designing appropriate fuzzy controllers $\mathbf{U}(\mathbf{t})$ in (8) and the fuzzy adaptation law of system parameters $\widetilde{\mathbf{a}}_{i}$ in (11), we are aiming to have

$$
\dot{\mathbf{V}}=-\mathbf{G e}(\mathbf{t})^{2} \leq 0 .
$$

In (12), the derivative of Lyapunov function is a negative semidefinite function of $\mathbf{e}(\mathbf{t})$. In current scheme of adaptive control of chaotic motion, traditional Lyapunov stability theorem and Barbalat lemma are used to prove the error vector approaches zero, as time approaches infinity. But the question, why the estimated or given parameters also approach the uncertain or goal parameters, remains with no answer. Through the pragmatically asymptotical stability theorem which is provided by Ge et al. in 1999 [18, 19], this question can be answered and addressed strictly.

\section{Novel Fuzzy-Modeling-Based Adaptive Synchronization}

In this section, two identical novel fuzzy Mathieu-Van der pol systems (M-V systems) with uncertainties are illustrated to show the effectiveness and feasibility of the proposed adaptive synchronization scheme. The M-V system is coupled with two typical nonlinear nonautonomous systems, Mathieu system and Van der pol system, which is a man-made complicated system for nonlinear study and has been proposed by Ge and Li [20] in 2009; this system can be described as follows:

$$
\begin{aligned}
& \dot{y}_{1}=y_{2} \\
& \dot{y}_{2}=-\left(a+b y_{3}\right) y_{1}-\left(a+b y_{3}\right) y_{1}^{3}-c y_{2}+d y_{3} \\
& \dot{y}_{3}=y_{4} \\
& \dot{y}_{4}=-e y_{3}+f\left(y_{4}-y_{3}^{2} y_{4}\right)+g y_{1},
\end{aligned}
$$

where $a, b, c, d, e, f, g$ are the system parameters. However, there are only three nonlinear terms in (13). In order to show the effectiveness of the proposed fuzzy adaptive control strategy in the following section, two uncertain terms are

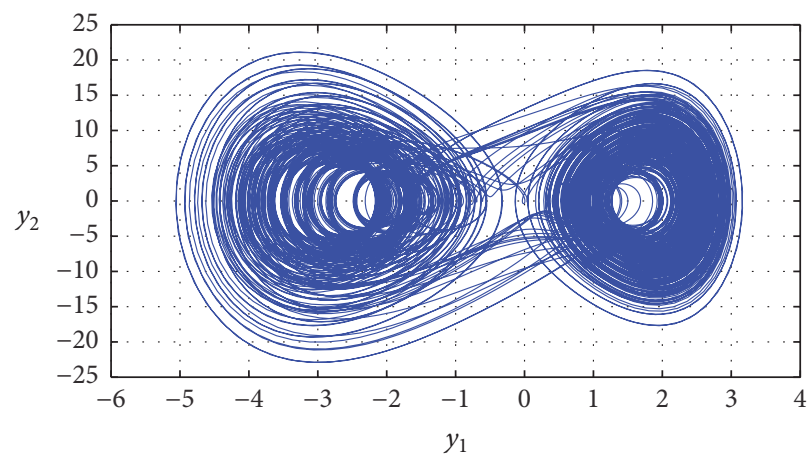

Figure 1: Chaotic motion of Mathieu-Van der pol system with uncertainties by $y_{1}-y_{2}$.

further added to system (13) to make the structure in (13) to be more complex, which is given as

$$
\begin{aligned}
& \dot{y}_{1}=y_{2}+\Delta_{1} \\
& \dot{y}_{2}=-\left(a+b y_{3}\right) y_{1}-\left(a+b y_{3}\right) y_{1}^{3}-c y_{2}+d y_{3} \\
& \dot{y}_{3}=y_{4}+\Delta_{2} \\
& \dot{y}_{4}=-e y_{3}+f\left(y_{4}-y_{3}^{2} y_{4}\right)+g y_{1},
\end{aligned}
$$

where $\Delta_{1}$ and $\Delta_{2}$ are uncertainties, and we defined that $\Delta_{1}=$ $\alpha_{1}\left(\sin w y_{2}\right)$ and $\Delta_{2}=\alpha_{2}\left(\sin w y_{4}\right)$, and then there are totally five nonlinear terms in this new system. This system exhibits chaos behavior with the parameters set as $a=10, b=3, c=$ $0.4, d=70, e=1, f=5, g=0.1, \alpha_{1}=5, \alpha_{2}=3, w=$ 0.1 and the initial states of system are $\left(y_{10}, y_{20}, y_{30}, y_{40}\right)=$ $(0.1,-0.5,0.1,-0.5)$. The complicated chaotic behaviors of the proposed dynamics system described in (14) are given in Figure 1.

Consider that the parameters $a, b, c, d, e, f, g, \alpha_{1} \alpha_{2}$ in (14) are all unknown; through the novel fuzzy modeling strategy, we have the following fuzzy M-V system, which is set as the master system:

$$
\dot{\tilde{\mathbf{Y}}}=\sum_{i=1}^{2} \widetilde{\mathbf{M}}_{i}\left(\mathbf{A}_{i} \mathbf{Y}(\mathbf{t})+\mathbf{B}_{i}\right)
$$

where

$$
\begin{aligned}
\mathbf{A}_{1} & =\left[\begin{array}{cccc}
0 & 1 & 0 & 0 \\
0 & -c & \left(d-b W_{2}\right) & 0 \\
0 & 0 & 0 & 1 \\
g & 0 & -e & 0
\end{array}\right], \\
\mathbf{B}_{1} & =\left[\begin{array}{c}
\alpha_{1} W_{1} \\
-a W_{2} \\
\alpha_{2} W_{3} \\
f W_{4}
\end{array}\right]
\end{aligned}
$$




$$
\begin{aligned}
& \mathbf{A}_{2}=\left[\begin{array}{cccc}
0 & 1 & 0 & 0 \\
0 & -c & \left(d+b W_{2}\right) & 0 \\
0 & 0 & 0 & 1 \\
g & 0 & -e & 0
\end{array}\right], \\
& \mathbf{B}_{2}=\left[\begin{array}{c}
-\alpha_{1} W_{1} \\
a W_{2} \\
-\alpha_{2} W_{3} \\
-f W_{4}
\end{array}\right] \\
& \widetilde{\mathbf{M}}_{1}=\left[\begin{array}{cccc}
M_{11} & 0 & 0 & 0 \\
0 & M_{21} & 0 & 0 \\
0 & 0 & M_{31} & 0 \\
0 & 0 & 0 & M_{41}
\end{array}\right], \\
& \widetilde{\mathbf{M}}_{2}=\left[\begin{array}{cccc}
M_{12} & 0 & 0 & 0 \\
0 & M_{22} & 0 & 0 \\
0 & 0 & M_{32} & 0 \\
0 & 0 & 0 & M_{42}
\end{array}\right],
\end{aligned}
$$

where $\dot{\mathbf{Y}}(\mathbf{t})=\left[\begin{array}{llll}\dot{y}_{1} & \dot{y}_{2} & \dot{y}_{3} & \dot{y}_{4}\end{array}\right]^{T}$ and initial conditions are set as $\left(y_{10}, y_{20}, y_{30}, y_{40}\right)=(0.1,-0.5,0.1,-0.5) . \mathbf{A}_{i}$ and $\mathbf{B}_{i}$ are the coefficient matrices with unknown parameters, and $\mathbf{M}_{i}$ are membership functions with unknown parameters and $\mathbf{M}_{1}+\mathbf{M}_{2}$ is identity matrix; the elements of the membership function matrices are fuzzy sets of each equation which are given as

$$
\begin{aligned}
& M_{11}=\frac{1}{2}\left(1+\frac{N_{1}}{W_{1}}\right), \\
& M_{12}=\frac{1}{2}\left(1-\frac{N_{1}}{W_{1}}\right), \\
& M_{21}=\frac{1}{2}\left(1+\frac{N_{2}}{W_{2}}\right), \\
& M_{22}=\frac{1}{2}\left(1-\frac{N_{2}}{W_{2}}\right), \\
& M_{31}=\frac{1}{2}\left(1+\frac{N_{3}}{W_{3}}\right), \\
& M_{32}=\frac{1}{2}\left(1-\frac{N_{3}}{W_{3}}\right), \\
& M_{41}=\frac{1}{2}\left(1+\frac{N_{4}}{W_{4}}\right), \\
& M_{42}=\frac{1}{2}\left(1-\frac{N_{4}}{W_{4}}\right),
\end{aligned}
$$

where $M_{11}+M_{12}=1, M_{21}+M_{22}=1, M_{31}+M_{32}=1$ and $M_{41}+M_{42}=1 . N_{1}=\sin w y_{2}, N_{2}=\left(y_{1}+y_{1}^{3}\right), N_{3}=\sin w y_{4}$, and $N_{4}=y_{4}-y_{3}^{2} y_{4}$ are the nonlinear terms groups and

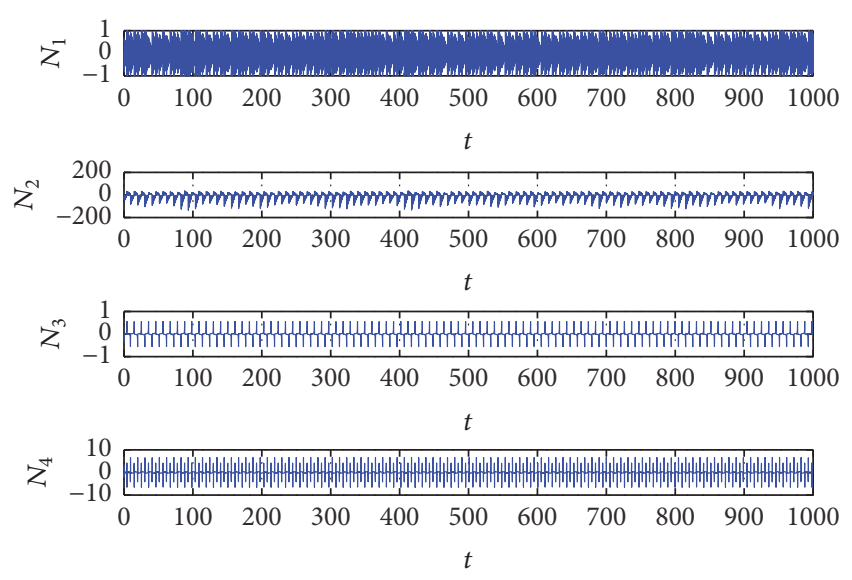

FIgURE 2: Time series of nonlinear terms groups in Mathieu-Van der pol system with uncertainties.

$\left(W_{1}, W_{2}, W_{3}, W_{4}\right)=(1,200,1,10)$ are the designed-boundary values, which is decided by the ranges of the nonlinear terms groups 1 to 4, and their time history is given in Figure 2.

In addition, a slave fuzzy M-V system with uncertainties and the designed fuzzy controllers as well as the estimated parameters are given as follows:

$$
\dot{\mathbf{X}}=\sum_{i=1}^{2} \widetilde{\mathbf{N}}_{i}\left(\widehat{\mathbf{A}}_{i} \mathbf{X}(\mathbf{t})+\widehat{\mathbf{B}}_{i}\right)+\mathbf{U}(\mathbf{t}),
$$

where

$$
\begin{aligned}
& \widehat{\mathbf{A}}_{1}=\left[\begin{array}{cccc}
0 & 1 & 0 & 0 \\
0 & -\widehat{c} & \left(\widehat{d}-\widehat{b} W_{2}\right) & 0 \\
0 & 0 & 0 & 1 \\
\widehat{g} & 0 & -\widehat{e} & 0
\end{array}\right], \\
& \widehat{\mathbf{B}}_{1}=\left[\begin{array}{c}
\widehat{\alpha}_{1} W_{1} \\
-\widehat{a} W_{2} \\
\widehat{\alpha}_{2} W_{3} \\
\widehat{f} W_{4}
\end{array}\right] \\
& \widehat{\mathbf{A}}_{2}=\left[\begin{array}{cccc}
0 & 1 & 0 & 0 \\
0 & -\widehat{c} & \left(\widehat{d}+\widehat{b} W_{2}\right) & 0 \\
0 & 0 & 0 & 1 \\
\widehat{g} & 0 & -\widehat{e} & 0
\end{array}\right] \text {, } \\
& \widehat{\mathbf{B}}_{2}=\left[\begin{array}{c}
-\widehat{\alpha}_{1} W_{1} \\
\widehat{a} W_{2} \\
-\widehat{\alpha}_{2} W_{3} \\
-\widehat{f} W_{4}
\end{array}\right]
\end{aligned}
$$




$$
\begin{aligned}
& \widetilde{\mathbf{N}}_{1}=\left[\begin{array}{cccc}
N_{11} & 0 & 0 & 0 \\
0 & N_{21} & 0 & 0 \\
0 & 0 & N_{31} & 0 \\
0 & 0 & 0 & N_{41}
\end{array}\right], \\
& \widetilde{\mathbf{N}}_{2}=\left[\begin{array}{cccc}
N_{12} & 0 & 0 & 0 \\
0 & N_{22} & 0 & 0 \\
0 & 0 & N_{32} & 0 \\
0 & 0 & 0 & N_{42}
\end{array}\right],
\end{aligned}
$$

where $\dot{\mathbf{X}}=\left[\begin{array}{llll}\dot{x}_{1} & \dot{x}_{2} & \dot{x}_{3} & \dot{x}_{4}\end{array}\right]^{T}$ and initial conditions are set as $\left(x_{10}, x_{20}, x_{30}, x_{40}\right)=(0.5,0.1,0.5,0.1) . \widehat{\mathbf{A}}_{i}$ and $\widehat{\mathbf{B}}_{i}$ are the coefficient matrices with estimated parameters. $\widehat{a}, \widehat{b}$, $\widehat{c}, \widehat{d}, \widehat{e}, \widehat{f}, \widehat{g}, \widehat{\alpha}_{1}, \widehat{\alpha}_{2}$ are the system estimated parameters and are given the initial values as $\widehat{a}_{0}=1, \widehat{b}_{0}=1, \widehat{c}_{0}=2, \widehat{d}_{0}=$ $65, \widehat{e}_{0}=8, \widehat{f}_{0}=1, \widehat{g}_{0}=-1, \widehat{\alpha}_{10}=1$, and $\widehat{\alpha}_{20}=1 . \widehat{\mathbf{N}}_{i}$ are membership functions and $\widetilde{\mathbf{N}}_{1}+\widetilde{\mathbf{N}}_{2}$ is identity matrix, and $\mathbf{U}(t)$ in (18) is designed as the fuzzy controller.

The error and error dynamics of the two identical nonlinear dynamics with unknown system parameters can be shown in the following form:

$$
\begin{aligned}
\mathbf{e}= & \mathbf{Y}-\mathbf{X}=\left[\begin{array}{l}
e_{1} \\
e_{2} \\
e_{3} \\
e_{4}
\end{array}\right]=\left[\begin{array}{l}
y_{1}-x_{1} \\
y_{2}-x_{2} \\
y_{3}-x_{3} \\
y_{4}-x_{4}
\end{array}\right] \\
\dot{\mathbf{e}}= & \dot{\mathbf{Y}}-\dot{\mathbf{X}} \\
= & \sum_{i=1}^{2} \widetilde{\mathbf{M}}_{i}\left(\mathbf{A}_{i} \mathbf{Y}(\mathbf{t})+\mathbf{B}_{i}\right)-\sum_{i=1}^{2} \widetilde{\mathbf{N}}_{i}\left(\widehat{\mathbf{A}}_{i} \mathbf{X}(\mathbf{t})+\widehat{\mathbf{B}}_{i}\right) \\
& -\mathbf{U}(\mathbf{t}) .
\end{aligned}
$$

Substituting the proposed fuzzy controllers $\mathbf{U}(\mathbf{t})$ described in Section 2 into the error dynamics in (20), we have the following simple form of error dynamic system:

$$
\dot{\mathbf{e}}(\mathbf{t})=\sum_{i=1}^{2} \widetilde{\mathbf{M}}_{i}\left(\widetilde{\mathbf{A}}_{i} \mathbf{Y}(\mathbf{t})+\widetilde{\mathbf{B}}_{i}\right)-\mathbf{G e}(\mathbf{t}),
$$

where the parameters errors are defined as $\widetilde{a}=a-\widehat{a}, \widetilde{b}=$ $b-\widehat{b}, \widetilde{c}=c-\widehat{c}, \widetilde{d}=d-\widehat{d}, \widetilde{e}=e-\widehat{e}, \tilde{f}=f-\widehat{f}, \widetilde{g}=$ $g-\widehat{g}, \widetilde{\alpha}_{1}=\alpha_{1}-\widehat{\alpha}_{1}$, and $\widetilde{\alpha}_{2}=\alpha_{2}-\widehat{\alpha}_{2}$ and $\mathbf{G} \in \mathfrak{R}^{4 \times 4}$ is designed as a positive-definite matrix given below; then we have the following adjustable control gains $\mathbf{K}_{1}$ and $\mathbf{K}_{2}$, which are interacted via the adaptation laws of parameters:

$$
\mathbf{G}=\left[\begin{array}{llll}
1 & 0 & 0 & 0 \\
0 & 1 & 0 & 0 \\
0 & 0 & 1 & 0 \\
0 & 0 & 0 & 1
\end{array}\right]
$$

$$
\begin{aligned}
& \mathbf{K}_{1}=\left[\begin{array}{cccc}
-1 & 1 & 0 & 0 \\
0 & -\widehat{c}-1 & \left(\widehat{d}-\widehat{b} W_{2}\right) & 0 \\
0 & 0 & -1 & 1 \\
\widehat{g} & 0 & -\widehat{e} & -1
\end{array}\right], \\
& \mathbf{K}_{2}=\left[\begin{array}{cccc}
-1 & 1 & 0 & 0 \\
0 & -\widehat{c}-1 & \left(\widehat{d}+\widehat{b} W_{2}\right) & 0 \\
0 & 0 & -1 & 1 \\
\widehat{g} & 0 & -\widehat{e} & -1
\end{array}\right] .
\end{aligned}
$$

Choose the candidate Lyapunov function mentioned in (10), and then we have the following square form of Lyapunov function:

$$
\begin{gathered}
\mathbf{V}\left(\mathbf{e}_{1}, \mathbf{e}_{2}, \mathbf{e}_{3}, \mathbf{e}_{4}, \widetilde{\mathbf{a}}, \widetilde{\mathbf{b}}, \widetilde{\mathbf{c}}, \widetilde{\mathbf{d}}, \widetilde{\mathbf{e}}, \widetilde{\mathbf{f}}, \widetilde{\mathbf{g}}, \widetilde{\alpha}_{1}, \widetilde{\alpha}_{2}\right)=\frac{1}{2}\left(\mathbf{e}(\mathbf{t})^{2}\right. \\
\left.+\sum_{i=1}^{k}\left(\mathbf{F}_{i} \widetilde{\mathbf{a}}_{i}^{2}\right)\right)=\frac{1}{2}\left(\mathbf{e}(\mathbf{t})_{1}^{2}+\mathbf{e}(\mathbf{t})_{2}^{2}+\mathbf{e}(\mathbf{t})_{3}^{2}+\mathbf{e}(\mathbf{t})_{4}^{2}\right. \\
\left.+\widetilde{\mathbf{a}}^{2}+\widetilde{\mathbf{b}}^{2}+\widetilde{\mathbf{c}}^{2}+\widetilde{\mathbf{d}}^{2}+\widetilde{\mathbf{e}}^{2}+\widetilde{\mathbf{f}}^{2}+\widetilde{\mathbf{g}}^{2}+\widetilde{\alpha}_{1}^{2}+\widetilde{\alpha}_{2}^{2}\right),
\end{gathered}
$$

where $\widetilde{\mathbf{a}}_{i}$ are the unknown parameters, $k=9$ is the number of unknown parameters, $\mathbf{F}_{i}$ refers to the adaptation gains where $\mathbf{F}_{i}>0$; in this case, all values of $\mathbf{F}_{i}$ are set as $\mathbf{F}_{i}=1$. Then, the derivatives of the Lyapunov function can be described as:

$$
\begin{aligned}
\dot{V}= & e(t)_{1} \dot{e}(t)_{1}+e(t)_{2} \dot{e}(t)_{2}+e(t)_{3} \dot{e}(t)_{3} \\
& +e(t)_{4} \dot{e}(t)_{4}+\tilde{a} \dot{\tilde{a}}+\tilde{b} \dot{\vec{b}}+\dot{\vec{c}}+\dot{\vec{d}}+\dot{e r e}+\tilde{f} \dot{\tilde{f}} \\
& +\tilde{g} \dot{\tilde{g}}+\widetilde{\alpha}_{1} \dot{\tilde{\alpha}}_{1}+\widetilde{\alpha}_{2} \dot{\tilde{\alpha}}_{2} .
\end{aligned}
$$

Substituting the error dynamic system in (25) into (24), we have

$$
\begin{aligned}
& \dot{V}=\left(M_{11}\left(\widetilde{\alpha}_{1} W_{1}\right) e_{1}+M_{12}\left(-\widetilde{\alpha}_{1} W_{1}\right) e_{1}-e_{1}^{2}\right) \\
& +\left(M_{21}\left(-\tilde{c} y_{2}+\left(\tilde{d}-\tilde{b} W_{2}\right) y_{3}-\tilde{a} W_{2}\right) e_{2}\right. \\
& \left.+M_{22}\left(-\widetilde{c} y_{2}+\left(\widetilde{d}+\widetilde{b} W_{2}\right) y_{3}+\widetilde{a} W_{2}\right) e_{2}-e_{2}^{2}\right) \\
& +\left(M_{31}\left(\widetilde{\alpha}_{2} W_{3}\right) e_{3}+M_{32}\left(-\widetilde{\alpha}_{2} W_{3}\right) e_{3}-e_{3}^{2}\right) \\
& +\left(M_{41}\left(\tilde{g} y_{1}-\tilde{e} y_{3}+\tilde{f} W_{4}\right) e_{4}\right. \\
& \left.+M_{42}\left(\tilde{g} y_{1}-\tilde{e} y_{3}-\tilde{f} W_{4}\right) e_{4}-e_{4}^{2}\right)+\tilde{a} \dot{\tilde{a}}+\tilde{b} \dot{\vec{b}}+\tilde{c} \dot{c} \\
& +\dot{\tilde{d}}+\dot{\tilde{e}} \dot{\tilde{e}}+\tilde{\tilde{f}} \dot{\tilde{f}}+\tilde{g} \dot{\tilde{g}}+\widetilde{\alpha}_{1} \dot{\tilde{\alpha}}_{1}+\widetilde{\alpha}_{2} \dot{\tilde{\alpha}}_{2} .
\end{aligned}
$$

According to the fuzzy adaptation laws of parameters proposed in Section 2, the adaptation laws can be designed as the following form with the membership functions of master system in (26), where the estimated parameter $\widehat{\alpha}_{1}$ is located in the first equation; therefore, it is arranged in pairs with 
the membership functions $M_{11}$ and $M_{12}$, and the estimated parameters $a, b$, and $c$ are included in the second equation; we arranged them to be in pairs with the membership functions $M_{21}$ and $M_{22}$, and the rest are arranged following the same strategy.

$$
\left[\begin{array}{c}
\dot{\widehat{\alpha}}_{1} \\
\dot{\hat{a}} \\
\dot{\hat{b}} \\
\dot{\hat{c}} \\
\dot{\tilde{d}} \\
\dot{\hat{\alpha}}_{2} \\
\dot{\hat{e}} \\
\dot{\hat{f}} \\
\dot{\hat{g}}
\end{array}\right]=\left[\begin{array}{c}
-\dot{\tilde{\alpha}}_{1} \\
-\dot{\tilde{a}} \\
\dot{\tilde{b}} \\
\dot{\tilde{c}} \\
\dot{\tilde{d}} \\
-\dot{\tilde{\alpha}} \\
-\dot{\tilde{e}} \\
-\dot{\tilde{f}} \\
-\dot{\tilde{g}}
\end{array}\right]
$$$$
=\left[\begin{array}{ccccccccc}
M_{11} & 0 & 0 & 0 & 0 & 0 & 0 & 0 & 0 \\
0 & M_{21} & 0 & 0 & 0 & 0 & 0 & 0 & 0 \\
0 & 0 & M_{21} & 0 & 0 & 0 & 0 & 0 & 0 \\
0 & 0 & 0 & M_{21} & 0 & 0 & 0 & 0 & 0 \\
0 & 0 & 0 & 0 & M_{21} & 0 & 0 & 0 & 0 \\
0 & 0 & 0 & 0 & 0 & M_{31} & 0 & 0 & 0 \\
0 & 0 & 0 & 0 & 0 & 0 & M_{41} & 0 & 0 \\
0 & 0 & 0 & 0 & 0 & 0 & 0 & M_{41} & 0 \\
0 & 0 & 0 & 0 & 0 & 0 & 0 & 0 & M_{41}
\end{array}\right]\left[\begin{array}{c}
W_{1} e_{1} \\
-W_{2} e_{2} \\
-W_{2} y_{3} \\
-y_{2} e_{2} \\
y_{3} e_{2} \\
W_{3} e_{3} \\
-y_{3} e_{4} \\
W_{4} e_{4} \\
y_{1} e_{4}
\end{array}\right]
$$

With the fuzzy adaptation law given in (26), we have the following result:

$$
\dot{V}=-e_{1}^{2}-e_{2}^{2}-e_{3}^{2}-e_{4}^{2} .
$$

The derivative of Lyapunov function in (27) is a negative semidefinite function of $\mathbf{e}$ and parameter differences. The Lyapunov asymptotical stability theorem is not satisfied. We cannot obtain that common origin of error dynamics and parameter dynamics is asymptotically stable. By pragmatical asymptotically stability theorem $[19,20], D$ is a 13 -manifold, $n=13$, and the number of error state variables $p=4$. When $e_{1}=e_{2}=e_{3}=e_{4}=0, \tilde{a}, \widetilde{b}, \widetilde{c}, \widetilde{d}, \widetilde{e}, \tilde{f}, \tilde{g}, \widetilde{\alpha}_{1}$, and $\widetilde{\alpha}_{2}$ take arbitrary values and $\dot{V}=0$, so $X$ is of 4 dimensions, $m=n-p=13-4=9, m+1<n$ is satisfied. According to the pragmatical asymptotically stability theorem, error vector $e$ approaches zero and the estimated parameters also approach the uncertain parameters. The equilibrium point is pragmatically asymptotically stable. Under the assumption of equal probability, it is actually asymptotically stable. The simulation results are shown in Figures 3, 4, and 5. 

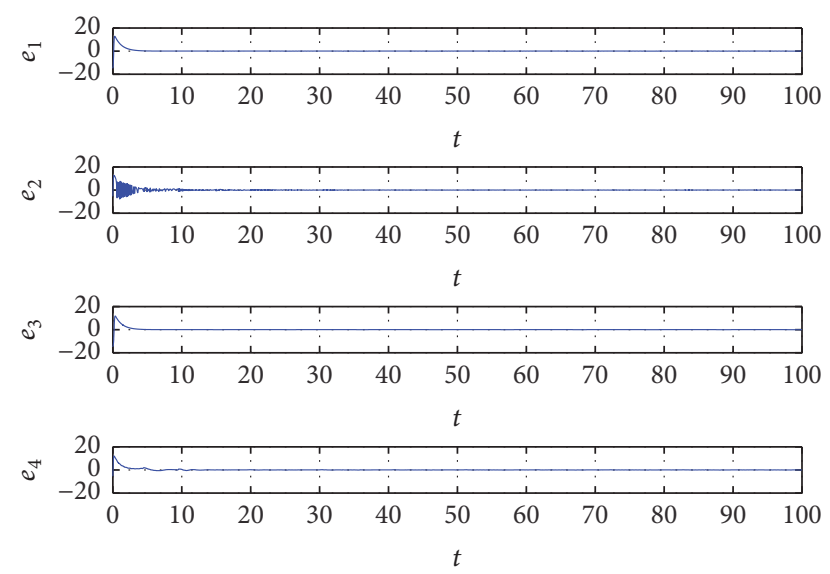

Figure 3: Time series of error states.
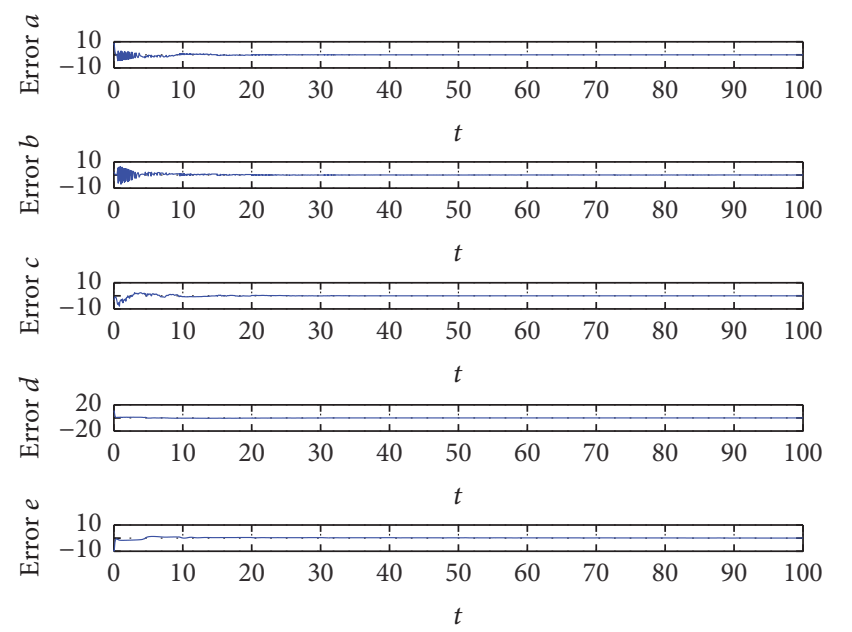

FIGURE 4: Time series of error of parameters I.

\section{Conclusions}

In this article, adaptive synchronization of advanced G-L fuzzy systems with parameters mismatch is achieved through the proposed adaptive control scheme. The final form of this advanced G-L fuzzy systems is the weighted sums of only two linear subsystems, and it provides us with a simpler way to analyze the stability of dynamics systems. Carrying the superiority of the proposed fuzzy model, a set of adaptive synchronization strategy is provided, which is designed to be composed of the adjustable control gains and the novel adaptation laws of parameters with fuzzy IF-THEN rules. The simulation results reveal that the proposed novel adaptive control strategy is effective and useful, and the parameters mismatch can be addressed simultaneously.

\section{Competing Interests}

The authors declare that there is no conflict of interests regarding the publication of this paper.
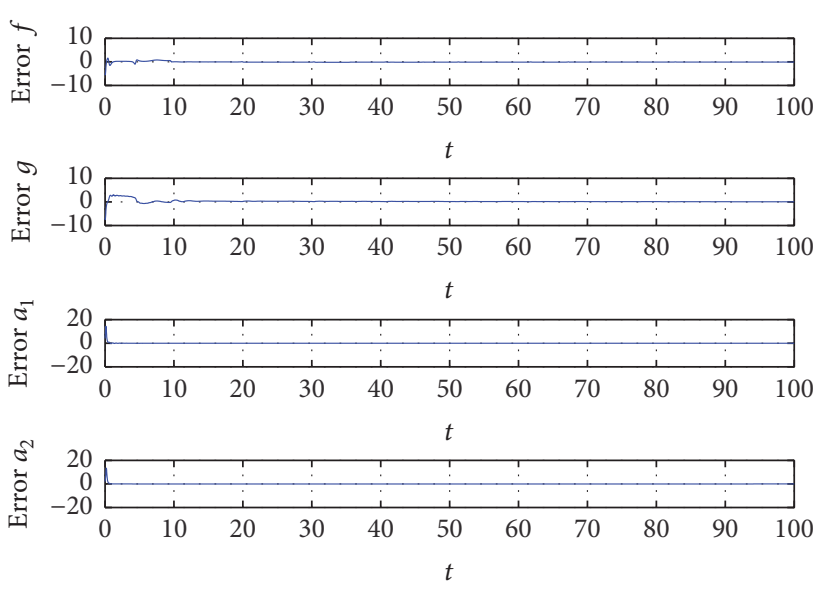

FIGURE 5: Time series of error of parameters II.

\section{Acknowledgments}

This study was funded in part by the Ministry of Science and Technology (MOST 105-2218-E-027-005), funded in part by MOST 104-2622-E-027-003- CC2, funded in part by MOST 105-2221-E-027-068 and NTUT-USTB-106-09, and funded in part by the Institute for the Development and Quality, Macao.

\section{References}

[1] L. A. Zadeh, "Fuzzy logic," IEEE Computers, vol. 21, no. 1, pp. 83-93, 1998.

[2] M. Khodaei Tehrani, A. Fereidunian, and H. Lesani, "Financial planning for the preventive maintenance of power distribution systems via fuzzy AHP," Complexity, vol. 21, no. 3, pp. 36-46, 2016.

[3] M. T. Hagh and N. Ghadimi, "Multisignal histogram-based islanding detection using neuro-fuzzy algorithm," Complexity, vol. 21, no. 1, pp. 195-205, 2015.

[4] N. Ghadimi, "A new hybrid algorithm based on optimal fuzzy controller in multimachine power system," Complexity, vol. 21, no. 1, pp. 78-93, 2015.

[5] H. Li, Z. Chen, L. Wu, H. Lam, and H. Du, "Event-triggered fault detection of nonlinear networked systems," IEEE Transactions on Cybernetics, pp. 1-12, 2016.

[6] T. Takagi and M. Sugeno, "Fuzzy identification of systems and its applications to modeling and control," IEEE Transactions on Systems, Man, and Cybernetics, vol. 15, no. 1, pp. 116-132, 1985.

[7] M. Kchaou and A. El-Hajjaji, "Resilient Ho sliding mode control for discrete-time descriptor fuzzy systems with multiple time delays," International Journal of Systems Science. Principles and Applications of Systems and Integration, vol. 48, no. 2, pp. 288-301, 2017.

[8] H. Li, J. Wang, and P. Shi, "Output-feedback based sliding mode control for fuzzy systems with actuator saturation," IEEE Transactions on Fuzzy Systems, vol. 24, no. 6, pp. 1282-1293, 2016.

[9] X.-L. Zhu, Y. Wang, and X. Du, "Stability criteria for continuous-time systems with additive time-varying delays," Optimal Control Applications \& Methods, vol. 35, no. 2, pp. 166-178, 2014.

[10] R. Rakkiyappan and N. Sakthivel, "Cluster synchronization for T-S fuzzy complex networks using pinning control with 
probabilistic time-varying delays," Complexity, vol. 21, no. 1, pp. 59-77, 2015.

[11] X.-L. Zhu, B. Chen, Y. Wang, and D. Yue, "Ho stabilization criterion with less complexity for nonuniform sampling fuzzy systems," Fuzzy Sets and Systems, vol. 225, pp. 58-73, 2013.

[12] S.-H. Tsai, "Robust $H_{\infty}$ stabilization conditions for a class of uncertain T-S fuzzy neutral systems with disturbance," Neurocomputing, vol. 193, pp. 68-80, 2016.

[13] H. Li, Z. Chen, Y. Sun, and H. Karimi, "Stabilization for a class of nonlinear networked control systems via polynomial fuzzy model approach," Complexity, vol. 21, no. 2, pp. 74-81, 2015.

[14] C.-S. Chen and M.-H. Lee, "Fuzzy neural network compensator for gantry stage synchronous motion," Applied Mechanics and Materials, vol. 145, pp. 234-239, 2012.

[15] X. Xie, D. Yue, T. Ma, and X. Zhu, "Further studies on control synthesis of discrete-time T-S fuzzy systems via augmented multi-indexed matrix approach," IEEE Transactions on Cybernetics, vol. 44, no. 12, pp. 2784-2791, 2014.

[16] Z.-M. Ge and S.-Y. Li, "Fuzzy modeling and synchronization of two totally different chaotic systems via novel fuzzy model," IEEE Transactions on Systems, Man, and Cybernetics, Part B: Cybernetics, vol. 41, no. 4, pp. 1015-1026, 2011.

[17] S.-Y. Li, L.-M. Tam, S.-E. Tsai, and Z.-M. Ge, "Novel fuzzy modeling and synchronization of chaotic systems with multinonlinear terms by advanced Ge-Li fuzzy model," IEEE Transactions on Cybernetics, vol. 46, no. 10, pp. 2228-2237, 2016.

[18] Z. M. Ge, J.-K. Yu, and Y.-T. Chen, "Pragmatical asymptotical stability theorem with application to satellite system," Japanese Journal of Applied Physics, Part 1, vol. 38, no. 10, pp. 6178-6179, 1999.

[19] Z.-M. Ge and J.-K. Yu, "Pragmatical asymptotical stability theorems on partial region and for partial variables with applications to gyroscopic systems," Journal of Mechanics, vol. 16, no. 4, pp. 179-187, 2000.

[20] Z.-M. Ge and S.-Y. Li, "Chaos control of new MAThieu-Van der Pol systems with new MAThieu-Duffing systems as functional system by GYC partial region stability theory," Nonlinear Analysis: Theory, Methods \& Applications, vol. 71, no. 9, pp. 4047-4059, 2009. 


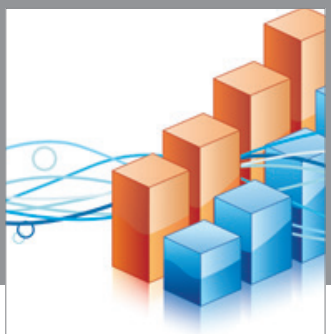

Advances in

Operations Research

vatem alat4

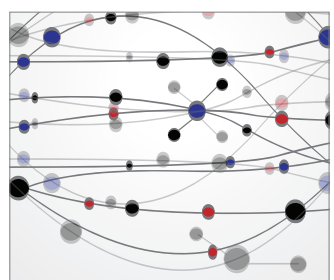

\section{The Scientific} World Journal
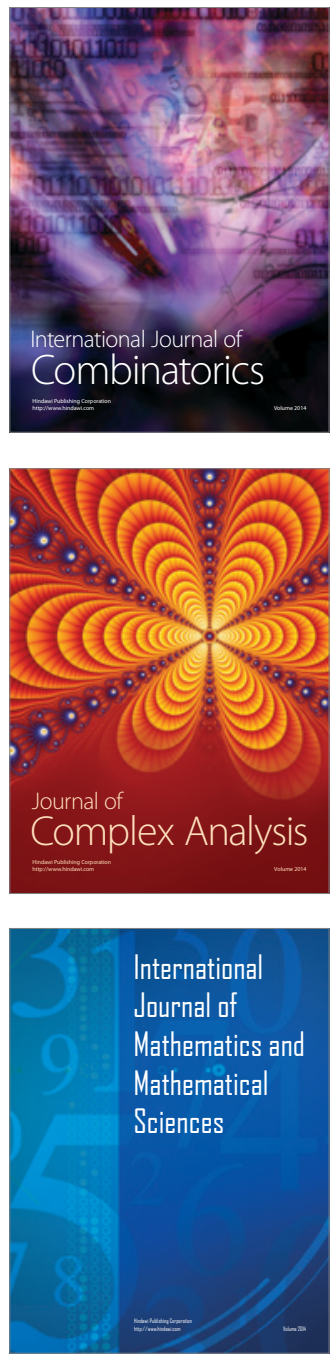
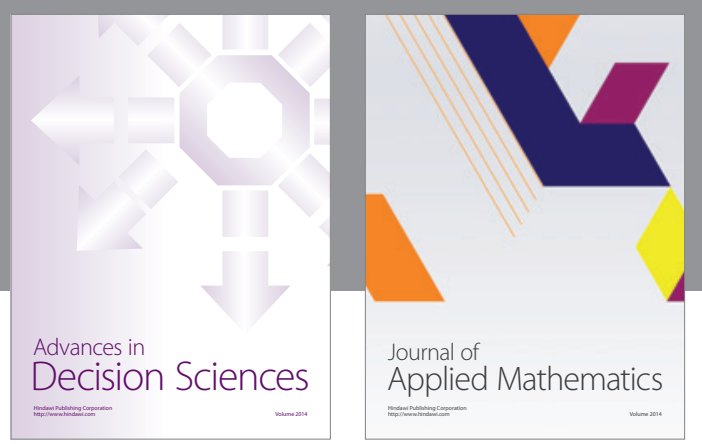

Algebra

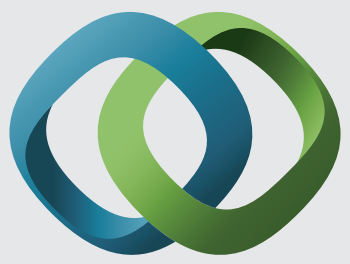

\section{Hindawi}

Submit your manuscripts at

https://www.hindawi.com
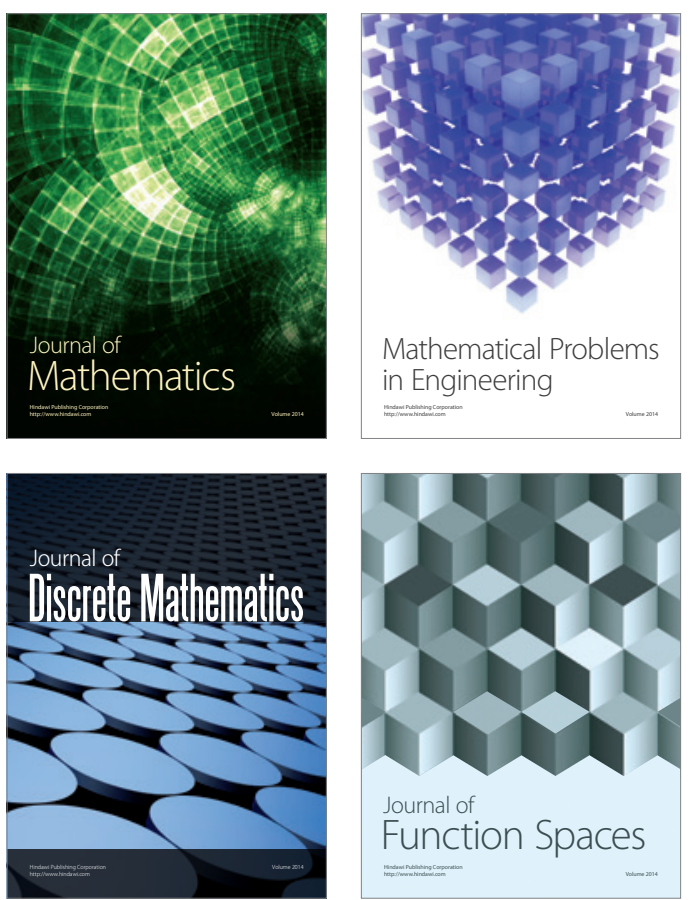

Mathematical Problems in Engineering
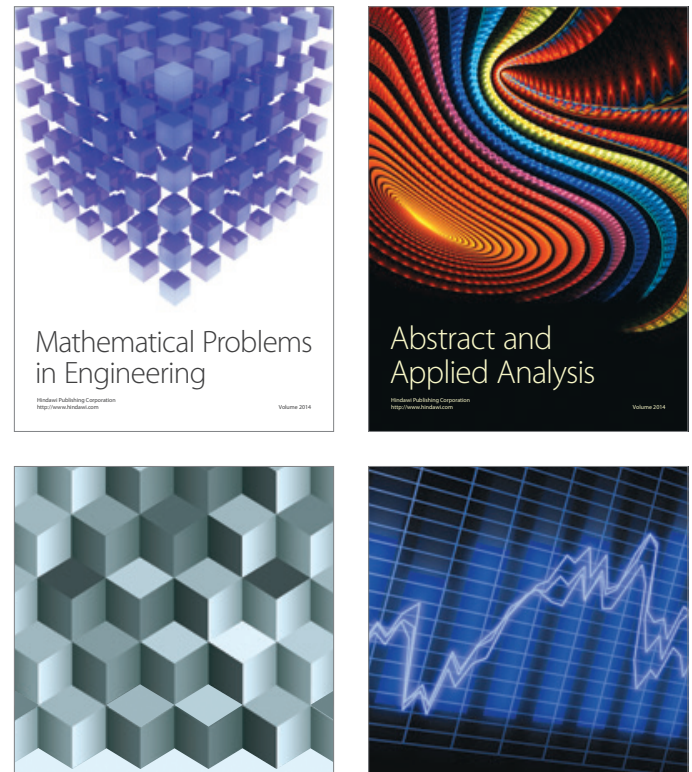

Journal of

Function Spaces

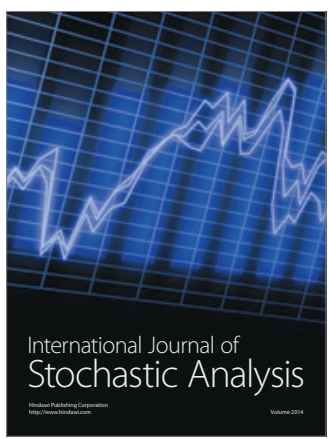

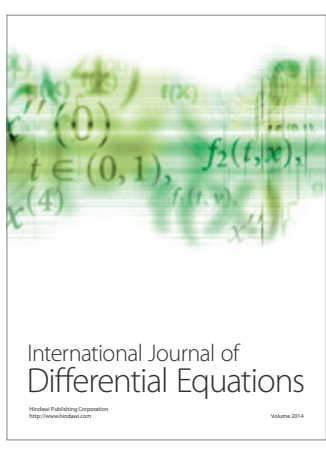
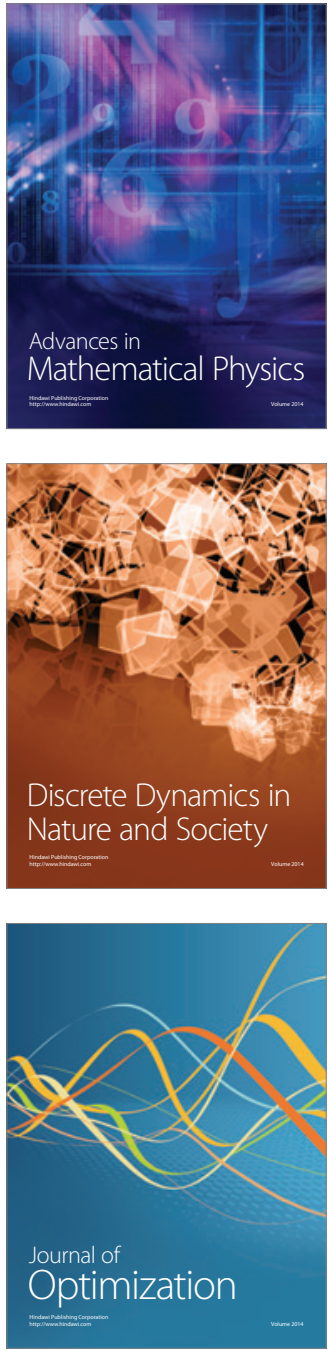